\title{
VALORACIÓN DEL ALUMNADO DEL GRADO DE EDUCACIÓN PRIMARIA ACERCA DE LA METODOLOGÍA DE APRENDIZAJE-SERVICIO
}

\author{
África María Cámara Estrella \\ Universidad de Jaén \\ María del Carmen Pegalajar Palomino \\ Universidad de Jaén
}

\begin{abstract}
Resumen. Este artículo analiza la valoración del alumnado sobre el desarrollo de competencias genéricas a partir de una metodología basada en el Aprendizaje-Servicio, frente al uso de estrategias metodológicas tradicionales. Dicho trabajo se ha realizado gracias a un Proyecto de Innovación Docente convocado por la Universidad de Jaén y centrado en el uso de la metodología de Aprendizaje-Servicio para el desarrollo de las prácticas en la asignatura "Sociedad, Familia y Escuela" del Grado de Educación Primaria. Para ello, se ha utilizado un estudio descriptivo, llevándose a cabo la recogida de datos mediante un cuestionario ad hoc $(\mathrm{n}=194)$. Los resultados revelan datos favorables para la metodología de AprendizajeServicio hacia el desarrollo de habilidades de investigación y de trabajo en grupo, así como el desarrollo de competencias instrumentales contempladas en los resultados de aprendizaje de la asignatura.
\end{abstract}

Palabras clave: Aprendizaje Social, innovaciones educativas, formación de competencias, comunidad educativa, educación superior.

\section{STUDENTS VALUATION OF PRIMARY EDUCATIONAL DEGREE ABOUT THE LEARNING-SERVICE METHODOLOGY}

\begin{abstract}
This article analyzes the evaluation of students on the development of generic competences based on a methodology based on Learning-Service, against the use of traditional methodological strategies. This work was carried out thanks to a Project of Teaching Innovation convened by the University of Jaen and focused on the use of the Learning-Service methodology for the development of practices in the subject "Society, Family and School" of the Education Degree Primary. For this purpose, a descriptive study was used, and data collection was carried out using an ad hoc questionnaire $(n=194)$. The results reveal favorable data for the Learning-Service methodology towards the development of research skills and group work as well as the development of instrumental competences contemplated in the learning outcomes of the subject.
\end{abstract}

Keywords: Social learning, educational innovation, skills training, educational community, higher education.

\section{AVALIAÇÃO DA ESTUDANTES DA CLASSE SOBRE A EDUCAÇÃO SERVICE-LEARNING METODOLOGIA}

Resumo. Este artigo analisa a avaliação dos alunos sobre o desenvolvimento de competências genéricas a partir de uma metodologia baseada em service-learning, sobre o uso de estratégias metodológicas 
tradicionais. Este trabalho foi possível graças a um projeto de ensino Inovação, organizado pela Universidade de Jaén e incidiu sobre o uso do serviço-learning metodologia para o desenvolvimento de práticas no assunto "Society, Família e Escola" Grau Educação primário. Para fazer isso, utilizou-se de um estudo descritivo, realizando a coleta de dados através de um questionário ad hoc ( $\mathrm{n}=194)$. Os resultados mostram dados favoráveis à metodologia de service-learning para o desenvolvimento de competências de investigação e de trabalho em grupo, bem como o desenvolvimento de competências instrumentais previstas nos resultados de aprendizagem do assunto.

Palavras-chave: Aprendizagem social, a inovação educacional, treinamento de habilidades, educação da comunidade, o ensino superior.

\section{Introducción}

La experiencia que se expone a continuación surge como consecuencia del Proyecto de Innovación Docente "El Aprendizaje-Servicio en la formación integral del alumnado de los Grados de Educación Infantil y Primaria", financiado por el Secretariado de Innovación Docente de la Universidad de Jaén. Dicha iniciativa ha desarrollado la metodología de Aprendizaje-Servicio vinculado a la asignatura "Sociedad, Familia y Escuela" impartida en el primer curso del Grado de Educación Primaria. Este proyecto tiene como objetivo que los alumnos perciban el AprendizajeServicio como una metodología capaz de hacerles competentes en su profesión, adquiriendo las competencias instrumentales, interpersonales y sistémicas, en comparación con una metodología más tradicional, basada en la reproducción de contenidos sin relación con el contexto educativo en el que han de desarrollarse.

Así pues, se considera la necesidad de abrir al alumnado nuevos horizontes en los que la educación no sea un simple instrumento de enseñanza, sino que también sea la oportunidad de transformar el entorno a través del desarrollo individual y colectivo, a través de aprendizajes interdisciplinares teóricos y prácticos, aprendiendo a la vez que se ofrece un servicio a la comunidad, en este caso, a un centro educativo concreto.

La transformación de los procesos de enseñanza y aprendizaje en todas las etapas educativas ha supuesto que la universidad se implique en un modelo de enseñanza más coherente y acorde con las demandas sociales y las necesidades de formación superior de los estudiantes. Este nuevo modelo educativo exige el desarrollo de un perfil profesional, de unos roles y unas actividades diferentes a las tradicionales en estudiantes y profesores de Educación Superior (Fernández, 2006); se centra en una concepción del aprendizaje constructivista, en la que el estudiante es el eje central en la creación de contenidos significativos, y el docente, mediador entre el conocimiento y el alumnado (Olmedo, 2013). Ello implica la revisión de los modelos de docencia tradicionales, requiriendo de un gran esfuerzo dinamizador e innovador por parte del profesorado y traduciéndose en una mejora del proceso de enseñanza-aprendizaje (Andreu, Sanz y Serrat, 2009; Iglesias, Toscano y Román, 2012). Se busca, en esta nueva metodología, dirigir los aprendizajes teóricos y prácticos a la formación para la vida, de concebir los aprendizajes como desarrollo de competencias (Díaz, 2014).

La enseñanza tradicional se centra en la inactividad del alumnado y la evaluación de conceptos y principios asimilados por los estudiantes, en contraposición con la enseñanza por competencias, que desarrolla habilidades, destrezas y comportamientos, que el alumnado ha de demostrar en el desempeño de su trabajo 
(López, 2011). Mientras que la enseñanza tradicional fomenta el individualismo, la repetición y la reproducción de contenidos, el uso de manuales únicos y la fragmentación de las materias, el modelo de formación por competencias potencia la interdisciplinariedad, el trabajo en grupo, la búsqueda y selección de información, la construcción de los propios aprendizajes, la implicación personal en los proyectos y la adquisición de capacidades personales (Imaz, 2015).

En este contexto, las universidades españolas han puesto en marcha cambios en las metodologías docentes y en los procedimientos de evaluación, orientándolos hacia la consecución de competencias en el alumnado (Carrasco et al., 2010; Cid, Zabalza y Doval, 2012; Zabalza, 2012). Así, se destaca como metodología innovadora de investigación y docencia, el Aprendizaje-Servicio (en adelante, ApS), como metodología capaz de combinar procesos de aprendizaje y de servicio a la comunidad en un solo proyecto, de modo que los estudiantes aprenden a trabajar según las necesidades reales del entorno para, de este modo, poder mejorarlo (Puig, Gijón, Martín y Rubio, 2011). Se trata de una forma de educación experiencial, en la que los alumnos se comprometen en actividades de ayuda a la comunidad, al tiempo que facilita el aprendizaje de una asignatura y el desarrollo de competencias profesionales (Rodríguez, 2014); supone una apertura a las necesidades sociales de la comunidad y proporciona un aprendizaje de conocimientos y valores de manera participativa y crítica (Torío, Peña y Hernández, 2014).

Esta comprensión del ApS en el ámbito educativo lleva implícita la consideración de cinco elementos configuradores, tales como (Martínez, 2005): el protagonismo del alumnado en el proceso de participación social y de aprendizaje académico, la atención a una necesidad real de la comunidad en la que se inserta la labor del alumnado, la conexión de la actividad de servicio comunitario con los objetivos curriculares de aprendizaje, la realización de un proyecto de servicio que será presentado a la comunidad y, finalmente, la reflexión y la realización de conexiones con los aprendizajes curriculares. El ApS es una herramienta pedagógica que facilita el desarrollo comunitario, de cohesión de la comunidad y compromiso responsable (López, 2010). Por ello, sirve como motor de superación de las dificultades, activación de redes sociales y fomento de la confianza de los ciudadanos (García y Mínguez, 2011).

Dicha propuesta define, por tanto, una de las metodologías innovadoras tendente a reforzar la calidad de la enseñanza universitaria, requiriendo de un modelo de intervención basado en el diálogo y la colaboración entre las personas "socias" participantes en todas las fases: planificación e iniciación de las sesiones de trabajo, control sobre cómo presentar y organizar las actividades, cómo dinamizar los trabajos en grupo o cómo evaluar su realización, apoyo a las personas participantes y sus contribuciones, información compartida, clarificación de objetivos, tareas, planificación y evaluación de la actuación del grupo en función de las propuestas y acciones realizadas (Lucas, 2012), valorando adecuadamente el logro de las competencias establecidas (Calvo y Mingorance, 2010). De este modo, el diálogo ayuda a identificar necesidades y buscar posibles respuestas desde la docencia y la investigación universitaria (Martínez y Folgueiras, 2015).

El ApS también cuenta con algunas limitaciones, como son la falta de tiempo para su programación y aplicación, el exceso de trabajo para ponerlo en práctica y evitar que se confunda con el voluntariado. Además, identificar los problemas a los que se puede ofrecer una solución desde las prácticas de las asignaturas universitarias, no es 
tarea fácil. Existe otra dificultad añadida, y es para la institución universitaria, algunos sectores sociales en los que se lleva a cabo el ApS, son realidades ajenas, y se presentan inconvenientes para su ejecución desde la misma organización (Martínez, 2010).

A pesar de todo, en el ámbito de la Educación Superior, se describe cómo el ApS es una buena estrategia metodológica, al combinar la formación teórica de una asignatura con la participación de los estudiantes en proyectos en los que se presta un servicio gratuito a la sociedad (Rodríguez, 2014); comprensión amplia y profunda de los contenidos del curso en que se realiza; desarrollo del sentido de compromiso ciudadano y mejora del conocimiento de sí mismo y su posición en la comunidad; fomento de la adquisición de competencias y habilidades difíciles de cumplir con otras metodologías. Estudios realizados por Álvarez, Rodríguez y San Fabián (2013) ponen de manifiesto la preferencia del alumnado hacia métodos de enseñanza más vinculados con la práctica (actividades de laboratorio, resolución de problemas, simulaciones y estudios de caso), siendo más beneficiosos cuando el docente clarifica desde el primer momento el procedimiento a seguir.

\section{El Aprendizaje-Servicio en la Universidad}

Por tanto, el ApS contribuye al desarrollo de las competencias profesionales específicas establecidas en el Marco Europeo de Educación Superior, al trabajar la enseñanza de las distintas titulaciones universitarias en diálogo con la comunidad, así como a las competencias transversales expuestas en el Proyecto Tunning (Tuning Education Structures in Europe):

- Competencias instrumentales: cognitivas, metodológicas, técnicas y lingüísticas.

- Competencias interpersonales: habilidades de relación social, integración en grupos, desarrollo de trabajos en equipos específicos y multidisciplinares.

- Competencias sistémicas: cualidades individuales, motivación para el trabajo, entendimiento, creatividad, adaptación, responsabilidad, iniciativa (Solanes, Núñez y Rodríguez, 2007).

Centrados en la formación del docente, el ApS constituye un recurso pedagógico de enorme valía: "a través de experiencias de aprendizaje en el servicio, los estudiantes de Magisterio aprender a ser profesionales reflexivos, sensibles a las cuestiones basadas en la comunidad" (Buchanan, Baldwin y Rudisill, 2002, p.30). El ApS, por tanto, fomenta profesionales competentes, con compromiso y empatía hacia los otros (Root, Callahan, y Billig2005). Los alumnos desarrollan competencias en planificación, comunicación con padres y representantes, mejora de la autoestima y autoeficacia, actitudes positivas en torno a la participación en actividades de la comunidad e incremento en el compromiso social (Anderson, 2000). Por su parte, trabajos realizados por Root, Callahan y Sepanski (2002) señalan la relación entre esta metodología y el desarrollo de competencias y disposiciones necesarias para la docencia eficaz, entre las que se destacan el desarrollo de la sensibilidad hacia el tema de la diversidad y el compromiso con la enseñanza.

El hecho de que el futuro docente participe en experiencias de ApS parece estar relacionado con su rol de promotor de tales actividades cuando ejerza la profesión (Bates, Drits, Allen y McCandless, 2009). Además, el contacto que ofrece la metodología de Aprendizaje-Servicio con la realidad y la posterior reflexión, estimula a los futuros maestros a examinar de forma crítica temas tales como el racismo y la igualdad de oportunidades (Hess, Laning y Vaughan, 2007), conduciendo al alumnado hacia el fomento del deber y la responsabilidad social (Annette, 2005; Billig, Jesse y Root, 2006). 
Esta metodología puede considerarse un recurso viable para proporcionar experiencias que facilitan en los futuros docentes la reflexión sobre los aspectos éticos de la profesión y su posicionamiento respecto a su labor profesional (Root, Callahan y Billig, 2005; Bell, Horn y Roxas, 2008); permite al estudiante involucrarse directamente con aquellos a quienes les ofrece un servicio, adaptándose a sus necesidades y a una realidad que, a menudo, es muy diferente a la que vive en el aula (Folgueiras, Luna y Puig, 2013). Además, proporciona al acompañante o mediador la posibilidad de la comprensión "in situ" de los problemas a los que se enfrentan los maestros a diario, a través de su vivencia (Mendia, 2013).

Inmersos en los programas formativos de los docentes, el ApS tiene el potencial de educar y comprometer a los estudiantes en debates críticos sobre temas relacionados con el poder, los privilegios y la justicia social y cómo estos temas se evidencian en los centros educativos (Morgan y Streb, 2001; Bender y Jordan, 2007; Ganley, Quintanar y Loop, 2007; Miller, Dunlap y González, 2007); sin duda, se trata de una herramienta poderosa que contribuye al desarrollo de competencias profesionales del docente y mejora el compromiso de los estudiantes con la comunidad y la justicia social (Aramburuzabala, 2012). No se trata de un "parche solidario" ante las desigualdades (García y Cotrina, 2015), sino un instrumento al servicio de la transformación que cuestiona el impacto de la propia universidad y su sentido.

Tales iniciativas guardan una estrecha relación con la creación de equipos de trabajo capaces de construir redes más amplias de colaboración. De este modo, la labor docente no debe entenderse como un camino solitario que lleve al cansancio y la frustración, sino como la generación de espacios de convivencia e intercambio que favorezcan un mayor dinamismo, motivación y satisfacción profesional (Rodríguez, 2007; Pacheco, 2008).

En definitiva, se trata de una propuesta metodológica innovadora, capaz de responder a los retos planteados en la sociedad actual en el nuevo marco europeo de Educación Superior, promoviendo una de las funciones básicas de la Universidad: formación de ciudadanos críticos, activos y responsables con su entorno (Amat y Moliner, 2010).

\section{Método}

Los objetivos específicos planteados para este trabajo pretenden:

- Comparar las percepciones del alumnado sobre la adquisición de competencias instrumentales mediante la ejecución de prácticas basadas en Aprendizaje-Servicio y prácticas tradicionales.

- Comparar las percepciones del alumnado sobre la adquisición de competencias interpersonales mediante la ejecución de prácticas basadas en Aprendizaje-Servicio y prácticas tradicionales.

- Comparar las percepciones del alumnado sobre la adquisición de competencias sistémicas mediante la ejecución de prácticas basadas en Aprendizaje-Servicio y prácticas tradicionales.

En el estudio que se presenta se ha utilizado un diseño metodológico basado en técnicas cuantitativas, a través de la encuesta descriptiva. El proyecto se dirige a alumnado del Grado de Educación Primaria que ha cursado la asignatura "Sociedad, Familia y Escuela" $(\mathrm{N}=300)$. Dicha asignatura se incluye en el plan de estudios de este 
Grado durante el $2^{\circ}$ cuatrimestre con una carga lectiva de 6 créditos ECTS; por ello, cuenta con 2 horas semanales de formación teórica y 1 hora de formación práctica.

La finalidad de la materia, de carácter básico, es proporcionar al alumnado las bases para comprender el carácter institucional del sistema educativo y el modo en que éste está relacionado con el medio y, especialmente, con la familia. De igual modo, se pretende concienciar al alumnado del fenómeno de la desigualdad en sus distintas manifestaciones y cómo ello afecta al hecho educativo.

Para el desarrollo de este trabajo, se ha contado con la colaboración de un centro educativo de la ciudad de Jaén (España), el cual ha aceptado a participar en dicho proyecto de innovación docente dada su disponibilidad para participar con la institución universitaria. Este centro cuenta con un total de 724 alumnos, distribuidos en 36 grupos: 9 de Educación Infantil, 8 de primer ciclo de Primaria, 7 de segundo ciclo de Primaria, 7 de tercer ciclo de Primaria, 5 unidades de apoyo a la integración y una unidad de compensación. El centro se caracteriza por ser bilingüe en lenguaje de signos, y por acoger a alumnado con diferentes necesidades educativas: autismo, deficiencias auditivas y visuales, de integración, etc. La Asociación de Madres y Padres de Alumnos (en adelante, AMPA), a su vez, impulsa actividades que se desarrollan en colaboración con diversos estamentos educativos, sociales y culturales (fundaciones benéficas, sociosanitarias, colegios, Universidad de Jaén, Patronato de deportes, Diputación provincial, etc.).

Concretamente, han sido 194 los participantes en el estudio, organizados en tres grupos (uno de mañana y dos de tarde), siendo éstos los que han participado en el desarrollo de las prácticas de las asignaturas y, además, han contestado al cuestionario facilitado una vez cursada la materia.

El instrumento de recogida de datos, elaborado ad hoc, está compuesto por 20 ítems distribuidos en tres dimensiones. Para su elaboración se ha tenido en cuenta la descripción de competencias a adquirir por el alumnado según el Proyecto Tunning: competencias instrumentales, interpersonales y sistémicas. Se trata de una escala tipo Likert cuyas opciones de respuesta oscilan entre 1 y 4 (siendo 1=nada, 2=algo, $3=$ bastante y $4=$ mucho), y en la que los alumnos deben señalar sus valoraciones acerca del nivel de adquisición de las competencias mediante la realización de prácticas tradicionales y prácticas con Aprendizaje-Servicio. La siguiente tabla muestra la relación de ítems que componen cada una de las dimensiones del instrumento, distribuidas según los tres tipos de competencias genéricas y el código para el cual han sido identificadas para su posterior análisis estadístico a través del programa estadístico SPSS (versión 19 para Windows):

\section{Tabla 1}

\section{Ítems del cuestionario}

\begin{tabular}{ll}
\hline \multicolumn{1}{c}{ Código } & Enunciado \\
\hline Competencias instrumentales \\
AT & Adquisición de contenidos teóricos \\
ADP & Adquisición de destrezas prácticas \\
DHI & Desarrollo de la habilidad intelectual \\
HRP & Habilidad de resolución de problemas
\end{tabular}




\begin{tabular}{ll}
\hline \multicolumn{1}{c}{ Código } & Enunciado \\
\hline Competencias interpersonales \\
M & Motivación sobre la necesidad del aprendizaje \\
TG & Desarrollo de trabajo grupal \\
HIP & Habilidades para la interacción personal, intelectual y emocional \\
CCE & Ver mi campo de estudio desde una perspectiva más amplia \\
PC & Habilidades de pensamiento crítico \\
HA & Habilidades de autocrítica \\
CE & Compromiso ético \\
ARS & Asumir responsabilidad con la sociedad \\
Competencias sistémicas \\
ATP & Aplicación de conocimientos teóricos a la realidad profesional \\
CRL & Comprensión de la realidad laboral posterior \\
HF & Desarrollo de habilidades para futuro profesional \\
C & Desarrollo de la creatividad \\
PR & Habilidades de pensamiento reflexivo \\
HI & Habilidades de investigación \\
HTA & Habilidades de trabajo autónomo \\
DDP & Diseño y dirección de proyectos de trabajo \\
\end{tabular}

Nota: Fuente: Proyecto Tunning.

El cuestionario ha sido sometido a un análisis de validez a partir de un juicio de expertos (validez de contenido), para comprobar que realmente los ítems del mismo son representativos de las dimensiones que se pretenden medir. Se envió la plantilla a tres jueces, expertos en el área de Didáctica y Organización Escolar, que valoraron en cada uno de los ítems los criterios de claridad, relevancia y coherencia, mediante una escala de 1 a 4 (siendo $1=$ nada y $4=$ mucho). El resultado ha sido que los jueces consideran que el cuestionario cumple estos criterios de claridad, relevancia y coherencia interna, con un acuerdo del $100 \%$.

Como anticipo al desarrollo de prácticas basadas en la metodología de Aprendizaje-Servicio, se han desarrollado una serie de actividades prácticas centradas en la metodología tradicional: lecciones magistrales, lecturas de textos, estudios de caso y prácticas guiadas, en las que el profesor expone el conocimiento al alumnado, teniendo éste un papel secundario en el proceso de enseñanza-aprendizaje. En este caso, los contenidos a tratar han estado relacionados en la educación familiar y la importancia de la escuela para el desarrollo del alumno.

Dichas prácticas están caracterizadas por un predomino de actividades de recepción y repetición de conocimientos, una organización de los alumnos en la que el docente se dirige al grupo total de la clase. Además, el profesorado ha evaluado la actividad a través de la asistencia del alumnado a clase.

El desarrollo del proyecto de Aprendizaje-Servicio ha requerido de una serie de fases consecutivas, elaboradas a partir de las indicaciones de Puig y Palos (2006), 
capaces de desarrollar un aprendizaje de calidad en el docente en formación, así como un auténtico servicio a la comunidad, tal como se indica en la tabla 2:

\section{Tabla 2}

Fases del proceso de ApS

\begin{tabular}{llc}
\hline \multicolumn{1}{c}{ FASES } & TEMPORALIZACIÓN \\
\hline 1. $\begin{array}{l}\text { Establecer las necesidades del entorno y posibles } \\
\text { servicios }\end{array}$ & Febrero 2015 \\
2. Establecer vínculo entre currículo y servicio & Febrero 2015 \\
3. Definir y organizar el proyecto & Febrero 2015 \\
4. Distribuir las tareas & Marzo 2015 \\
5. Obtener información & Marzo 2015 \\
6. Ejecutar el servicio & Marzo-Mayo 2015 \\
7. Valorar los resultados del servicio & Junio 2015 \\
8. Proyectar acciones futuras & Junio 2015 \\
& & Septiembre 2015 \\
\hline
\end{tabular}

Nota: Fuente: Elaboración propia.

En primer lugar, se contactó con el Equipo directivo del centro con el que se iba a colaborar, fijándose un primer encuentro. En esta primera toma de contacto entre los responsables del proyecto de innovación con los equipos docentes y una representación del AMPA, se comunicó el deseo de desarrollar el Proyecto de Innovación Docente en el centro, planteando los objetivos del mismo, así como las actividades y recursos que los alumnos del Grado de Educación Primaria podían aportar a la enseñanza y funcionamiento del centro.

Una representación de la AMPA manifestó su deseo de colaborar también en el proyecto, por lo que se programó un seminario en la Universidad con los estudiantes y la Junta directiva de la Asociación, con el fin de que se diesen a conocer a los alumnos, explicasen las actividades que llevaban a cabo a lo largo del año, y expusieran sus necesidades. Motivaron a los alumnos a participar en la elaboración de programas formativos de Escuelas de Padres. También pidieron colaboración en la organización de actividades culturales y lúdicas dirigidas a la comunidad educativa. Tanto en el caso de la Escuela de Padres como en las actividades culturales y lúdicas, los universitarios adquirieron el compromiso de, además de diseñar los materiales necesarios, desarrollarlas en el centro, cuando la dirección del AMPA así lo requiriera.

A raíz de esta sesión, se inició la segunda fase del proceso de AprendizajeServicio: el establecimiento de relaciones entre el currículum de la asignatura "Sociedad, familia y Escuela" y su vinculación con el servicio que se prestaría al centro. En esta fase, se ha realizado una explicación teórica de la metodología del ApS, haciendo hincapié en la idea de que los aprendizajes adquiridos se refuerzan si se comunican, y que uno de los fines de la educación es transformar la sociedad en una comunidad más justa e igualitaria. Además, y para que los alumnos tuvieran la 
información de la asignatura, se publicó la guía docente de la misma en la web de la Facultad, ofreciéndose las indicaciones precisas para que fuesen adquiriendo información sobre los temas de estudio de las mismas.

En las fases siguientes, se conformaron los grupos de trabajo entre los universitarios (máximo 5 personas) quienes eligieron la temática que abordarían en su trabajo práctico y se planificaron las sesiones de clase de manera que, durante las mismas, los grupos realizasen el trabajo bajo la supervisión de la profesora, que resolvía las dudas técnicas y ajustaba los proyectos a las necesidades que se habían planteado y que están indicados más arriba. Cada grupo buscó información sobre el tema a desarrollar, contextualizando así la propuesta que se haría al centro. Además, se facilitó a los alumnos un esquema de trabajo para el diseño de las propuestas formativas, tras lo cual cada grupo pasó a realizar el proyecto en grupo.

Dichos trabajos debían incluir la siguiente información:

- Introducción

- Justificación de la temática

- Análisis del contexto a nivel de centro y aula

- Desarrollo de actividades

- Temporalización

- Diseño de materiales para el desarrollo de las actividades

- Evaluación

Una vez los trabajos estuvieron realizados, se expusieron en clase para darlos a conocer al resto de compañeros. En esta sesión, se invitó a los alumnos a cumplimentar un cuestionario con la intención de comparar sus percepciones sobre la adquisición de competencias en las prácticas tradicionales desarrolladas al inicio de la asignatura, y las desarrolladas con esta metodología, con el fin de conocer si hay diferencias entre ambas.

Por último, y una vez evaluados los alumnos, se organizó con la dirección del centro y del AMPA una visita al centro para presentar los trabajos realizados. En esta visita, se ofrecieron al AMPA los materiales elaborados por los alumnos, mediante una exposición de los mismos, confeccionados por los propios alumnos, en que explicaron qué habían hecho y cómo respondían a las demandas efectuadas por los padres. En el desarrollo de esta visita, los miembros de la Junta directiva del AMPA, pidieron a los alumnos que, para continuar/completar el servicio, participaran en la organización de las actividades deportivas y solidarias que realizaban los padres, tales como carreras solidarias, tardes de juegos, convivencias de las familias del centro, etc.

Con esta actividad, los estudiantes observaron cómo con sus prácticas habían vinculado los aprendizajes a contextos educativos concretos, plasmando así sus conocimientos en hechos tangibles, y haciendo realidad que la teoría se hace práctica, y la práctica se nutre de la teoría, enriqueciéndose mutuamente.

\section{Resultados}

Este trabajo presenta la valoración del alumnado del Grado de Educación Primaria de la Universidad de Jaén acerca de la adquisición de las competencias propias de la materia "Sociedad, Familia y Escuela" mediante el desarrollo de la metodología ApS, frente al uso de metodologías tradicionales. Los datos se han organizado, para su presentación, según la clasificación de competencias de tipo instrumental, interpersonal 
y sistémico elaboradas de acuerdo con el planteamiento europeo en la enseñanza universitaria. De este modo, se han tomado como referencia las puntuaciones medias para cada uno de los ítems de cada dimensión, estableciendo una comparativa entre las prácticas tradicionales y las prácticas basadas en una metodología de ApS.

\section{Tabla 3}

Análisis descriptivo de las competencias instrumentales

\begin{tabular}{lcccc}
\hline & \multicolumn{2}{c}{ APS } & \multicolumn{2}{c}{ TRADICIONAL } \\
& M & DT & M & DT \\
\hline Adquisición de contenidos teóricos & 3.2 & 0.21 & 2.3 & 0.26 \\
Adquisición de destrezas prácticas & 3.1 & 0.31 & 2.4 & 0.37 \\
Desarrollo de la habilidad intelectual & 3.0 & 0.22 & 2.7 & 0.21 \\
Habilidad de resolución de problemas & 3.0 & 0.37 & 2.5 & 0.15 \\
\hline
\end{tabular}

De manera global, tal y como se muestra en la tabla 1 , los alumnos consideran que con las prácticas basadas en $\mathrm{ApS}$, son capaces de adquirir las competencias instrumentales en mayor medida que con las prácticas tradicionales. El desarrollo de esta metodología les facilita, en mayor medida que las prácticas tradicionales, la adquisición de conocimientos teóricos $(\mathrm{AT}=3,2$ vs 2,3$)$ y destrezas prácticas $(\mathrm{ADP}=3,1$ vs 2,4$)$. En esta última variable se aprecia mayor diferencia en las valoraciones de los alumnos, mientras que en la adquisición de contenidos teóricos encuentran los mejores resultados. Consideran, además, que el ApS potencia el desarrollo de la habilidad intelectual (DHI=3, vs 2,7) más que las prácticas tradicionales, así como la habilidad de resolución de problemas y toma de decisiones ( $\mathrm{HRP}=3$ vs 2,5$)$.

\section{Tabla 4}

Análisis descriptivo de las competencias interpersonales

\begin{tabular}{lcccc}
\hline & \multicolumn{3}{c}{ APS } & \multicolumn{2}{c}{ TRADICIONAL } \\
& M & DT & M & DT \\
\hline Motivación sobre la necesidad del aprendizaje & 3.2 & 0.23 & 2.5 & 0.10 \\
Desarrollo de trabajo grupal & 3.4 & 0.12 & 2.7 & 0.15 \\
Habilidades para la interacción personal, intelectual y y & 3.2 & 0.20 & 2.6 & 0.22 \\
emocional & & & & \\
Ver mi campo de estudio desde una perspectiva más & 3.2 & 0.21 & 2.5 & 0.30 \\
amplia & & & & \\
Habilidades de pensamiento crítico & 3 & 0.23 & 2.5 & 0.27 \\
Habilidades de autocrítica & 3.1 & 0.21 & 2.6 & 0.26 \\
Compromiso ético & 3.1 & 0.20 & 2.5 & 0.20 \\
Asumir responsabilidad con la sociedad & 3.4 & 0.15 & 2.6 & 0.14 \\
\hline
\end{tabular}

Centrados en el desarrollo de las competencias interpersonales en el alumnado, (tabla 2), se observa cómo el desarrollo de la habilidad de pensamiento crítico ( $\mathrm{PC}=3$, vs 2,5$)$, autocrítica $(\mathrm{HA}=3,1$ vs 2,6$)$ y compromiso ético $(\mathrm{CE}=3,1$ vs 2,5$)$ son valorados de manera favorable, frente al uso de la metodología tradicional. No obstante, el desarrollo del trabajo grupal $(\mathrm{TG}=3,4$ vs 2,7$)$ y la asunción de responsabilidad social 
(ARS $=3,4$ v s 2,6) son las variables mejor valoradas por el alumnado, siendo en esta última donde se aprecia mayor diferencia en el uso del ApS y la metodología tradicional. Los alumnos se muestran más motivados con la metodología de ApS que con la tradicional ( $M=3,2$ vs 2,5), encontrándose también diferencias importantes en el uso de ambas metodologías en las habilidades para la interacción personal, intelectual y emocional (HIP=3,2 vs 2,6) así como en la ampliación del campo de estudio ( $\mathrm{CCE}=3,2$ vs 2,5$)$.

\section{Tabla 5}

Análisis descriptivo de las competencias sistémicas

\begin{tabular}{lccccc}
\hline & & \multicolumn{2}{c}{ APS } & \multicolumn{2}{c}{ TRADICIONAL } \\
& & M & DT & M & DT \\
\hline $\begin{array}{l}\text { Aplicación de conocimientos teóricos a la realidad } \\
\text { profesional }\end{array}$ & 3.1 & 0.22 & 2.4 & 0.21 \\
Comprensión de la realidad laboral posterior & & & & \\
Desarrollo de habilidades para futuro profesional & 3.1 & 0.36 & 2.5 & 0.10 \\
Desarrollo de la creatividad & 3.2 & 0.15 & 2.5 & 0.35 \\
Habilidades de pensamiento reflexivo & 3.2 & 0.14 & 2.5 & 0.42 \\
Habilidades de investigación & 3.1 & 0.36 & 2.7 & 0.35 \\
Habilidades de trabajo autónomo & 3.3 & 0.20 & 2.7 & 0.20 \\
Diseño y dirección de proyectos de trabajo & 3.1 & 0.17 & 2.7 & 0.20 \\
\hline
\end{tabular}

En la valoración de las competencias sistémicas (tabla 3), los datos indican que el diseño y dirección de proyectos de trabajo constituyen la variable mejor valorada por los estudiantes para la metodología de ApS (DDP=3,3), así como las habilidades de investigación $(\mathrm{HI}=3,3)$, en comparación con el desarrollo de estas competencias en las prácticas tradicionales (2,7 y 2,7 respectivamente). Los alumnos consideran que la creatividad se potencia en mayor medida con el ApS $(\mathrm{C}=3,2$ vs 2,5$)$, así como el desarrollo de habilidades para el futuro profesional $(\mathrm{HF}=3,2$ vs 2,5$)$ y la aplicación de conocimientos teóricos en la realidad profesional (ATP=3,1 vs 2,4). Estas variables son las que muestran mayores diferencias entre las metodologías comparadas.

Finalmente, se observa que los alumnos aprecian menos diferencias en las variables de pensamiento reflexivo y de trabajo autónomo ( $\mathrm{PR}=3,1$ vs 2,7 y $\mathrm{HTA}=3,1$ vs 2,7$)$, mientras que la variable de comprensión de la realidad laboral posterior es la menos valorada para la metodología de $\mathrm{ApS}(\mathrm{CRL}=3,1$ vs 2,5).

\section{Discusión y conclusiones}

En las reflexiones realizadas tras la fase de evaluación del proyecto, se han aportado resultados favorables por parte de los alumnos. En general, se observa cómo los estudiantes del Grado de Educación Primaria, tras cursar la asignatura "Sociedad, familia y Escuela", han valorado de forma positiva la adquisición de las competencias genéricas establecidas en el proyecto Tunning; de manera especial, se destaca la adquisición de contenidos teóricos, asunción de responsabilidad social, trabajo en grupo, dirección y diseño de proyectos de trabajo y habilidades de investigación. Estos resultados coinciden con la aportación de Batlle (2011), quién afirma cómo la metodología del ApS favorece el sentido social de la educación e integra los aspectos 
cognitivos, actitudinales y morales del aprendizaje en el alumnado mediante la adquisición de conocimientos básicos para el ejercicio de la profesión, resolución de conflictos y toma de decisiones, así como la capacitación para trabajar en equipo y asunción de responsabilidades sociales. Además, favorece la adquisición de competencias y habilidades difíciles de cumplir con otras metodologías (Rodríguez (2014); se trata, por tanto, de una metodología vinculada a las preferencias del alumnado universitario (Álvarez, Rodríguez y San Fabián, 2013), al tener un marcado carácter práctico.

Con esta experiencia innovadora, los alumnos se han acercado a la realidad de los centros educativos para conocer y comprender las necesidades que la comunidad educativa demanda. Entrar en contacto con la realidad educativa y social de un centro, ha permitido que los estudiantes conozcan de primera mano las actividades que se realizan, el funcionamiento del AMPA, como asociación participativa vital del centro, y ha redundado en su enriquecimiento personal y profesional.

Reflexionando sobre el proceso de aprendizaje en la formación inicial del docente, la metodología basada en el Aprendizaje-Servicio, que articula en un solo proyecto la teoría y la práctica social, anima al alumnado a profundizar en su formación y compartir los conocimientos que va adquiriendo a lo largo de su carrera con aquellos sectores de la sociedad que así lo requieran. Con esta metodología, los alumnos se ven más eficaces en el desempeño de su trabajo y mejoran las conductas prosociales, mejor que son el desempeño de prácticas universitarias tradicionales.

Centrados en el ámbito de la responsabilidad social, los alumnos participantes han manifestado su deseo de continuar colaborando con el centro educativo, al margen de las asignaturas, por lo que se entiende que poner al servicio de otros el conocimiento adquirido, lleva al alumnado a implicarse en servicios a la comunidad. Conocer las necesidades del AMPA del centro, les ha alentado para trabajar en el aula universitaria, al conocer que su trabajo va a ser tenido en cuenta, va a traspasar las paredes del aula y va a llegar a sus destinatarios reales, en este caso, los padres, siendo los propios alumnos los encargados de llevarlos a la práctica.

Además, el proyecto ha supuesto un importante apoyo para el centro educativo y, más concretamente, para el AMPA, quien ha comprobado cómo desde la Universidad se puede dar respuesta a sus necesidades y demandas, formándose equipos de trabajo capaces de participar y colaborar en las acciones que desde esta entidad puedan desarrollarse. No obstante, la metodología planteada ha supuesto un cambio radical de la función docente en Educación Superior, adoptando éste el rol de facilitador de contextos y materiales que mejoren las condiciones de aprendizaje del alumnado. Así pues, se han establecido mecanismos de reflexión capaces analizar la práctica docente planteada en la formación inicial del docente, valorando de forma positiva la metodología de ApS como experiencia de buenas prácticas en el marco de la Educación Superior. A pesar de suponer un trabajo extra, les ha permitido implicarse aún más con los alumnos y los trabajos realizados, saliendo del entorno de la universidad para observar la realidad escolar y conocer sus necesidades, adecuando las prácticas de la asignatura a las mismas. Para ello, se ha requerido de un trabajo de coordinación y planificación continua entre el profesorado, el centro educativo y los estudiantes, generando un clima de mayor confianza basado en un diálogo más igualitario y sincero (Aubert, Flecha, García, Flecha y Racionero, 2009).

En relación con los objetivos propuestos en el estudio, se puede decir que los estudiantes universitarios participantes valoran en mayor medida la adquisición de 
competencias instrumentales, interpersonales y sistémicas, a través de las prácticas de ApS, que con las prácticas tradicionales.

En definitiva, se trata de un proyecto favorable para todos y cada uno de los agentes que, de un modo u otro, han participado en el mismo, bien a través de la aplicación del conocimiento teórico a la realidad educativa, mediante el desarrollo de prácticas innovadoras en Educación Superior o, simplemente, dando a conocer la realidad escolar y aceptando su participación en las propuestas formativas elaboradas por los alumnos. Esta experiencia basada en el Aprendizaje-Servicio ha revelado la necesidad de revisar los modelos de docencia tradicionales en el profesorado universitario en pro de una metodología participativa, formativa y práctica en el que el alumnado pueda adquirir el conocimiento sobre su futura actividad profesional a partir del contacto directo con la realidad educativa, dando respuesta a las necesidades y demandas de los agentes implicados en la educación.

No obstante, se trata de una experiencia concreta vinculada al desarrollo de trabajos prácticos para una asignatura en el Grado de Educación Primaria, lo cual dificulta la generalización de los resultados a otras muestras. Además, se han tenido algunas dificultades para ajustar la organización de la experiencia según el calendario académico de ambas instituciones educativas, lo que ha impedido la puesta en práctica y posterior desarrollo de las propuestas didácticas elaboradas por el alumnado.

Así pues, y de cara a futuros trabajos de investigación, se pretende ampliar la muestra objeto de estudio y desarrollar experiencias de ApS para la realización de actividades prácticas en otras asignaturas propias del Grado de Educación Infantil y Primaria y así comparar las percepciones del alumnado de ambas titulaciones. De igual modo, podría resultar interesante conocer la percepción de los docentes universitarios acerca de las potencialidades y limitaciones de este tipo de metodología, así como las dificultades que encuentra su puesta en práctica en las aulas universitarias. Además, se pretende ampliar la metodología de la investigación, llevando a cabo análisis cualitativos basados en entrevistas y grupos de discusión que permitan analizar en profundidad las percepciones de los agentes implicados acerca del desarrollo de tal estrategia metodológica en el ámbito de la Educación Superior.

En este sentido, se apuesta por el desarrollo de un servicio a la comunidad a través del ApS, como metodología motivadora, que favorece no solo la implicación social sino también el aprendizaje de competencias del alumnado. No obstante, se entiende que se trata de una experiencia piloto desarrollada en una asignatura del Grado de Educación Primaria de la Universidad de Jaén, por lo que, para futuros trabajos, se propone seguir utilizando esta propuesta con otras materias vinculadas a la formación inicial del docente, ampliándose a otros centros educativos y otras necesidades, pues el ApS abarca diferentes ámbitos de actuación en la comunidad educativa.

\section{Referencias}

Álvarez, E., Rodríguez, A. y San Fabián, J.L. (2013). Metodologías y recursos didácticos en los títulos de Grado. ¿Qué hace el profesorado y qué quiere el alumnado? Tendencias pedagógicas, 22, 127-147.

Amat, F. y Moliner, L. (2010). El Aprendizaje Servicio en la Universidad: una estrategia en la formación de la ciudadanía crítica. Revista Electrónica Interuniversitaria de Formación del Profesorado, 13, 69-77. 
Anderson, J. (2000). Learning in deed: Service-learning and preserviceteachereducation. ECS IssuePaper. Education Commission of the States: Denver.

Andreu, L., Sanz, M. y Serrat, E. (2009). Una propuesta de renovación metodológica en el marco del Espacio Europeo de Educación Superior: los pequeños grupos de investigación cooperativos. REIFOP, Revista Electrónica Interuniversitaria de Formación del Profesorado, 12(3), 111-126.

Annette, J. (2005). Character, civic renewal and service learning for democratic citizenship in higher education. British Journal of EducationalStudies, 53(3), 326-340.

Aramburuzabala, P. (2012). El aprendizaje-servicio en la formación de maestros. Comunicación presentada en el VII CIDUI: La Universidad: una institución de la sociedad. Barcelona.

Aubert, A., Flecha, A., García, C., Flecha, R. y Racionero, S. (2009). Aprendizaje dialógico en la sociedad de la información. Barcelona: Hipatia.

Bates, A.K., Drits, D., Allen, C. y McCandless, P. (2009). Service learning as an instructional strategy for the preparation of teachers. TheJournal of EffectiveTeaching, 9, 5-23.

Batlle, R. (2011) ¿De qué hablamos cuando hablamos de Aprendizaje-Servicio?.Crítica, 972, 1-6.

Bell, C., Horn, B. y Roxas, K. (2008). We know it's service, but what are they learning? Preservice teachers' understandings of diversity. Equity \& Excellence in Education, 40(2), 123-133.

Bender, G. y Jordan, R. (2007). Student perceptions and attitudes about Community Service-Learning in the teacher training curriculum. South African Journal of Education, 27, 631-654.

Billig, S., Jesse, D. y Root, S. (2006). The impact of service-learning in high school students civic engagement. Evaluation report prepared for the Carnegie Corporation of New York. Denver, CO: RMC Research Corporation.

Buchanan, A.M., Baldwin, S.C. y Rudisill, M.E. (2002). Service learning as scholarship in teacher education. EducationalResearcher, 31(5), 30-36.

Calvo, A. y Mingorance, A.C. (2010). Evaluación continua de conocimientos vs de competencias: Resultados de la aplicación de dos métodos valorativos diferentes. Revista de Investigación Educativa, 28(2), 361-383.

Carrasco, A.; Donoso, J.A.; Duarte, T.; Hernández, J.J.; López, R. y Núñez, C. (2010). La utilización de metodologías activas de aprendizaje en la enseñanza de la contabilidad. XIV Encuentro de la Asociación Española de Profesores Universitarios de Contabilidad: La Coruña.

Cid, A.; Zabalza, M.A. y Doval, M.I. (2012). La docencia universitaria: un modelo para su análisis. Revista de Docencia Universitaria, 10(1), 87-104.

Díaz, A. (2014) Construcción de programas de estudio en la perspectiva del enfoque del desarrollo de competencias. Perfiles Educativos, 143, 142-162.

Fernández, M. (2006). Metodologías activas para la formación de competencias. Educatio siglo XXI, 24, 35-56. 
Folgueiras, P., Luna, E. y Puig, G. (2013). Aprendizaje-Servicio: estudio del grado de satisfacción de estudiantes universitarios. Revista de Educación, 362, 159-185.

Furco, A. y Billing, S. H. (2002). Service-Learning. The Essence of the Pedagogy. Greenwich: Information Age Publishing.

Ganley, D.D., Quintanar, A.P. y Loop, L.S. (2007). Raising the Bar of Teacher Quality: Accountability, Collaboration and Social Justice. CollegeTeachingQuarterly, 10(3), 1-11.

García, A. y Mínguez, R. (2011). Los límites de la educación en valores cívicos: cuestiones y propuestas pedagógicas. Educación XXI, 14(2), 263-285.

García, M. y Cotrina, M.J. (2015). El Aprendizaje-Servicio en la formación inicial del profesorado: de las prácticas educativas críticas a la institucionalización curricular. Profesorado. Revista de currículum y formación del profesorado, 19, $1-25$.

Hess, D.J., Lanig, H. y Vaughan, W. (2007). Educating for equity and social justice: A conceptual model for cultural engagement. Multicultural Perspectives, 9, 32-39.

Iglesias, J., Toscano, M.O. y Román, C. (2012). Taller de prensa económica como instrumento de innovación educativa. Revista de Investigación educativa, 30(2), 423-440.

Imaz, J.I. (2015). Aprendizaje basado en proyectos en los grados de Pedagogía y Educación Social: ¿Cómo ha cambiado tu ciudad?.Revista Complutense de Educación, 26(3), 679-696.

López Herrerías, J.A. (2010). Educación de calidad y en competencias para la competitividad o para la cooperación. Revista Complutense de Educación, 21(1), 107-122.

López Ruiz, J.I. (2011). Un giro copernicano en la enseñanza universitaria: formación por competencias. Revista de Educación, 356, 279-301.

Lucas, S. (2012). Aprendizaje-Servicio como propuesta de integración curricular del Voluntariado en la Responsabilidad Social Universitaria. Ponencia presentada a las II Jornadas sobre Responsabilidad Social: Valladolid: Universidad de Valladolid y Caja de Burgos.

Martínez, M. y Folgueiras, P. (2015). Evaluación participativa, Aprendizaje-Servicio y Universidad. Profesorado. Revista de currículum y formación del profesorado, 19, 128-143.

Martínez, M. (ed.) (2010) Aprendizaje servicio y responsabilidad social de las universidades. Barcelona: Ediciones Octaedro-Ice.

Martínez-Odría, A. (2005). Service-Learning o Aprendizaje-Servicio: una propuesta de incorporación curricular del voluntariado. Tesis Doctoral. Universidad de Navarra.

Mendia, R. (2013). Aprendizaje-Servicio solidario: el acompañamiento educativo. Bilbao: Zerbikas.

Miller, K.K., Dunlap, C.Z. y González, A. (2007). The impact of a freshman year community-based service-learning experience on the achievement of standards articulated for teacher candidates. The School Community Journal, 2, 111-121. 
Morgan, W. y Streb, M. (2001). Building citizenship: How quality service-learning develops civic values. Social SciencesQuarterly, 1, 154-169.

Olmedo, E.V. (2013). Enfoques de aprendizaje de los estudiantes y metodología docente: Evolución hacia el nuevo sistema de formación e interacción propuesta en el EEES. Revista de Investigación Educativa, 31 (2), 429-445.

Pacheco, J.L. (2008). Los grupos de trabajo de profesores como estrategia de mejora. Profesorado. Revista de Currículum y Formación del profesorado, 2(1), 1-9.

Puig J. Mª Gijón, M; Martín, X. y Rubio, L. (2011) Aprendizaje-servicio y Educación para la Ciudadanía. Revista de Educación, número extraordinario 2011, 45-67.

Puig, J. M. y Palos, J. (2006). Rasgos pedagógicos del Aprendizaje-Servicio. Cuadernos de Pedagogía. 357, 60-63.

Rodríguez, J.M. (2007). Espacios de reflexión y de actuación asociados al quehacer educativo del profesorado. Revista de Ciencias de la Educación, 210, 205-218.

Rodríguez, M. (2014). El Aprendizaje-Servicio como estrategia metodológica en la Universidad. Revista Complutense de Educación, 25, 95-113.

Root, S., Callahan, J. yBillig, S. (2005). The National Service-Learning in Teacher Education Partnership: A Research Retrospective. Grenwich: Information Age.

Root, S., Callahan, J. y Sepanski, J. (2002). Building teaching dispositions and servicelearning practice: a multi-site study. Michigan Journal of Community ServiceLearning, 8, 50-60.

Solanes, A., Núñez, R. y Rodríguez, J. (2007). Elaboración de un cuestionario para la evaluación de competencias genéricas en estudiantes universitarios. Apuntes de psicología, 8(1), 35-49.

Torío, S., Peña, J.V. y Hernández, J. (2014). Aprendizaje Servicio como entrenamiento al emprendimiento social: una experiencia universitaria. Procedia-social and Behavioral Sciences, 139, 504-511.

Tuning, P. (2006). Tunning Educational Structures in Europe.Disponible en http://www.unideusto.org/tuningeu/images/stories/Publications/Tuning_brochur e_en_espanol_listo.pdf.

Zabalza, M.A. (2012). Las competencias en la formación del profesorado: de la teoría a las propuestas prácticas. Tendencias Pedagógicas, 20, 5-32.

Fecha de recepción: 10/10/2016

Fecha de revisión: 06/02/2017

Fecha de aceptación: 12/02/2017 